\title{
Increasing cocoa butter-like lipid production of Saccharomyces cerevisiae by expression of selected cocoa genes
}

\author{
Yongjun Wei ${ }^{1,2}$, Michael Gossing ${ }^{1,2}$, David Bergenholm ${ }^{1,2}$, Verena Siewers ${ }^{1,2}$ and Jens Nielsen ${ }^{1,2,3^{*}}$
}

\begin{abstract}
Cocoa butter (CB) extracted from cocoa beans mainly consists of three different kinds of triacylglycerols (TAGs), 1,3-dipalmitoyl-2-oleoyl-glycerol (POP, C16:0-C18:1-C16:0), 1-palmitoyl-3-stearoyl-2-oleoyl-glycerol (POS, C16:0C18:1-C18:0) and 1,3-distearoyl-2-oleoyl-glycerol (SOS, C18:0-C18:1-C18:0), but CB supply is limited. Therefore, CB-like lipids (CBL, which are composed of POP, POS and SOS) are in great demand. Saccharomyces cerevisiae produces TAGs as storage lipids, which are also mainly composed of C16 and C18 fatty acids. However, POP, POS and SOS are not among the major TAG forms in yeast. TAG synthesis is mainly catalyzed by three enzymes: glycerol-3-phosphate acyltransferase (GPAT), lysophospholipid acyltransferase (LPAT) and diacylglycerol acyltransferase (DGAT). In order to produce CBL in S. cerevisiae, we selected six cocoa genes encoding GPAT, LPAT and DGAT potentially responsible for $\mathrm{CB}$ biosynthesis from the cocoa genome using a phylogenetic analysis approach. By expressing the selected cocoa genes in S. cerevisiae, we successfully increased total fatty acid production, TAG production and CBL production in some S. cerevisiae strains. The relative CBL content in three yeast strains harboring cocoa genes increased 190, 230 and $196 \%$ over the control strain, respectively; especially, the potential SOS content of the three yeast strains increased 254,476 and $354 \%$ over the control strain. Moreover, one of the three yeast strains had a 2.25 -fold increased TAG content and 6.7-fold higher level of CBL compared with the control strain. In summary, CBL production by S. cerevisiae were increased through expressing selected cocoa genes potentially involved in CB biosynthesis.
\end{abstract}

Keywords: Phylogenetic analysis, TAG biosynthetic genes, Cocoa butter-like lipids, Metabolic engineering, Synthetic biology, Cell factories

\section{Introduction}

Cocoa butter (CB) extracted from cocoa seeds (Theobroma cacao) mainly contains three different kinds of triacylglycerols (TAGs)-1,3-dipalmitoyl-2-oleoyl-glycerol (POP, C16:0-C18:1-C16:0), 1-palmitoyl-3-stearoyl2-oleoyl-glycerol (POS, C16:0-C18:1-C18:0) and 1,3-distearoyl-2-oleoyl-glycerol (SOS, C18:0-C18:1C18:0), which are composed of C16 and C18 fatty acids (Jahurul et al. 2013). CB is mainly used for chocolate production and there is a world-wide increasing demand for chocolate (Clough et al. 2009). However, the CB supply

\footnotetext{
*Correspondence: nielsenj@chalmers.se

1 Department of Biology and Biological Engineering, Chalmers University of Technology, SE-41296 Gothenburg, Sweden

Full list of author information is available at the end of the article
}

is limited because cocoa trees only grow in a limited geographical zone and diseases affect cocoa trees nearly every year (Clough et al. 2009). Therefore, finding new sustainable sources for $\mathrm{CB}$ ingredients, such as $\mathrm{CB}$-like lipids (CBL, which are composed of POP, POS and SOS), is of interest. TAGs are used as energy and carbon storage in yeasts (Sorger and Daum 2003), such as the model Saccharomyces cerevisiae and the main components of its TAGs are usually C16 and C18 fatty acids (Koch et al. 2014; Sorger and Daum 2003), suggesting that S. cerevisiae has the potential to be used for CBL production.

Triacylglycerol is an ester composed of one glycerol and three fatty acids, and its synthesis is mainly catalyzed by three different kinds of enzymes: glycerol-3-phosphate acyltransferase (GPAT), lysophospholipid acyltransferase (LPAT) and diacylglycerol acyltransferase 
(DGAT), which can add acyl-coenzyme As (acyl-CoAs) to the $s n-1, s n-2$ and $s n-3$ position of glycerol, respectively (Chapman and Ohlrogge 2012). S. cerevisiae contains two GPATs (Gpt2p and Sct1p), two LPATs (Slc1p and Slc4p) and one DGAT (Dgalp). Additionally, another phospholipid:diacylglycerol acyltransferase, PDAT (Lro1p), can also synthesize TAG using diacylglycerol (DAG) and phospholipid as substrates (Fig. 1) (Coleman and Lee 2004; de Kroon et al. 2013; Ratledge 2002; Zheng and Zou 2001). Previous studies showed that double deletions of either the two GPAT genes or the two LPAT genes of $S$. cerevisiae were lethal, indicating these genes are essential in yeast (Benghezal et al. 2007; Zheng and Zou 2001). Though C16:0, C16:1, C18:0 and C18:1 are the four main fatty acids in the total fatty acid composition of S. cerevisiae (Khoomrung et al. 2012), only small amounts of CBL (POP, POS, SOS) have been identified among the TAGs in wild-type S. cerevisiae cells (Ejsing et al. 2009), suggesting that its GPAT, LPAT and DGAT enzymes might not be optimal for CBL production.

As natural $\mathrm{CB}$ or its equivalents are mainly derived from plant fruits or seeds, global analyses of some plant GPAT, LPAT and DGAT genes, could reveal potential CB biosynthetic genes (Jahurul et al. 2013). However, different from yeast, plants usually contain many GPAT, LPAT and DGAT genes. For example, there are ten GPAT genes, nine LPAT genes and four DGAT genes in the genome of the model plant Arabidopsis thaliana (Chen et al. 2011; Kim et al. 2005; Turchetto-Zolet et al. 2011). The GPAT, LPAT and DGAT genes of $A$. thaliana are diverse (Chen et al. 2011; Kim et al. 2005) and some of them are functionally redundant, such as GPAT4 and GPAT8. Only a double knockout of both GPAT4 and GPAT8 can strongly reduce cutin biosynthesis in $A$. thaliana (Li et al. 2007). It is therefore difficult to select GPAT, LPAT and DGAT genes specific for $\mathrm{CB}$ biosynthesis directly from plants. As genome information of T. cacao is available (Argout et al. 2011; Motamayor et al. 2013), recovering GPAT, LPAT and DGAT genes of $T$. cacao, which are potentially responsible for $\mathrm{CB}$ biosynthesis, and expressing them in S. cerevisiae might provide functional information and at

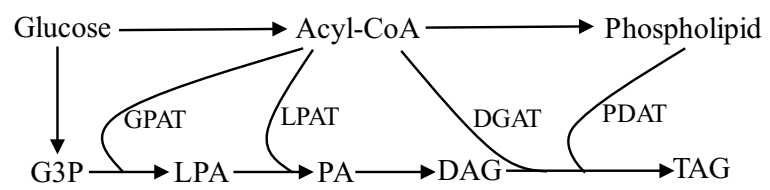

Fig. 1 Three Enzymes, GPAT, LPAT, DGAT, determine the TAG structure in the TAG biosynthetic pathway. G3P glycerol-3-phosphate, LPA lysophosphatidic acid, PA phosphatidic acid, DAG diacylglycerol, TAG triacylglycerol, GPAT glycerol-3-phosphate acyltransferase, LPAT lysophosphatidic acid acyltransferase, DGAT acyl-CoA: diacylglycerol acyltransferase, PDAT phospholipid: diacylglycerol acyltransferase the same time enable improved CBL production in this yeast. Two DGAT genes of T. cacao have been characterized and described by Zhang (Zhang 2012), and their expression in a yeast strain deficient in TAG synthesis led to accumulation of TAGs different from the wild-type strain.

Here we retrieved six potential CB biosynthetic genes (two GPAT, two LPAT and two DGAT genes) in T. cacao using a phylogenetic analysis approach. In order to verify the function of these cocoa genes and to understand their effects on lipid production of S. cerevisiae, we expressed them individually or combined in $S$. cerevisiae and compared the total fatty acid content in the engineered yeasts. Based on the total fatty acid results, we selected three strains harboring cocoa genes for further analysis of the total lipid composition and the TAG profile, and compared these with the similar measurements for the control strain. This analysis provided new functional insight into $\mathrm{CB}$ biosynthetic enzymes and advanced CBL production using S. cerevisiae as a cell factory.

\section{Materials and methods \\ Strains and plasmids}

The Escherichia coli strain DH5 $\alpha$ was used for all the cloning work in this study, and the E. coli transformants were selected on LB medium containing $100 \mu \mathrm{g} \mathrm{ml}{ }^{-1}$ ampicillin. The $S$. cerevisiae strain used was CEN.PK 11311C (MATa MAL2-8c SUC2 ura3-52 his3- $\Delta 1$ ), which was kindly provided by Kötter Entian and Kötter (2007). The strains harboring cocoa genes were constructed based on S. cerevisiae CEN.PK 113-11C and all the yeast strains constructed in this study are listed in Table 1. Yeast strains harboring cocoa genes were selected on synthetic complete (SC) dropout media (Formedium Ltd) (Li et al. 2015). The minimal medium, containing $7.5 \mathrm{~g} \mathrm{l}^{-1}$ $\left(\mathrm{NH}_{4}\right)_{2} \mathrm{SO}_{4}, 14.4 \mathrm{~g} \mathrm{l}^{-1} \mathrm{KH}_{2} \mathrm{PO}_{4}, 0.5 \mathrm{~g} \mathrm{l}^{-1} \mathrm{MgSO}_{4} \cdot 7 \mathrm{H}_{2} \mathrm{O}$, $20 \mathrm{~g} \mathrm{l}^{-1}$ glucose, trace metal solution and vitamin solution (Verduyn et al. 1992), supplemented with $100 \mathrm{mg} \mathrm{l}^{-1}$ histidine, was used for $20 \mathrm{ml}$ shake flask batch cultivation. The nitrogen-limited medium (named NLM medium in the text) was used for 11 shake flask batch cultivations (Yang et al. 2014).

Phylogenetic analysis of cocoa GPAT, LPAT and DGAT genes The GPAT, LPAT and DGAT gene sequences of T. cacao annotated by CGD (Cacao Genome Database, http:// www.cacaogenomedb.org/) and KEGG databases were downloaded from the Genbank database (Kanehisa et al. 2016). Reference GPAT, LPAT and DGAT sequences of A. thaliana, Homo sapiens and S. cerevisiae were directly downloaded from the KEGG database (Kanehisa et al. 2016). Multiple alignments of amino acid sequences of GPATs, LPATs or DGATs were carried out using the 
Table 1 List of strains derived from S. cerevisiae CEN.PK 113-11C and used in this study

\begin{tabular}{|c|c|c|}
\hline Name & Expression plasmids & Properties \\
\hline YJO & pBS01A & Empty vector \\
\hline YJ-G01 & pYJ-G01 & TCGPAT1 expression \\
\hline YJ-G02 & pYJ-G02 & TCGPAT2 expression \\
\hline YJ-L01 & pYJ-L01 & TCLPAT1 expression \\
\hline YJ-LO2 & pYJ-LO2 & TCLPAT2 expression \\
\hline YJ-D01 & pYJ-D01 & TCDGAT1 expression \\
\hline YJ-D02 & pYJ-D02 & TCDGAT2 expression \\
\hline YJ-111 & pYJ-111 & TCGPAT1, TCLPAT1 and TCDGAT1 gene combination expression \\
\hline YJ-112 & pYJ-112 & TCGPAT1, TCLPAT1 and TCDGAT2 gene combination expression \\
\hline YJ-121 & pYJ-121 & TCGPAT1, TCLPAT2 and TCDGAT1 gene combination expression \\
\hline YJ-122 & pYJ-122 & TCGPAT1, TCLPAT2 and TCDGAT2 gene combination expression \\
\hline YJ-211 & pYJ-211 & TCGPAT2, TCLPAT1 and TCDGAT1 gene combination expression \\
\hline YJ-212 & pYJ-212 & TCGPAT2, TCLPAT1 and TCDGAT2 gene combination expression \\
\hline YJ-221 & pYJ-221 & TCGPAT2, TCLPAT2 and TCDGAT1 gene combination expression \\
\hline YJ-222 & pYJ-222 & TCGPAT2, TCLPAT2 and TCDGAT2 gene combination expression \\
\hline
\end{tabular}

MAFFT online version (Katoh and Standley 2013). The alignment results were used to create phylogenetic trees using the MEGA 6.06 software, and the used method was the Neighbor-Joining method with Poisson correction (Tamura et al. 2013). The bootstrap confidence values were based on 1000 replicates. The pair wise-deletion option was used to treat gaps in the alignment of GPAT, LPAT or DGAT sequences. Two cocoa GPAT, two cocoa LPAT and two cocoa DGAT genes which were similar to characterized TAG biosynthetic genes were selected as potential $\mathrm{CB}$ biosynthetic genes and used for expression in $S$. cerevisiae.

\section{Synthesis of cocoa genes and expression plasmid construction}

Six cocoa genes encoding GPAT, LPAT or DGAT were synthesized codon-optimized for expression in S. cerevisiae (GeneArt Gene Synthesis, Thermo Fisher Scientific). The six synthesized cocoa gene sequences were deposited at the GenBank database under the accession number of KX982578-KX982583.

The primers used to amplify cocoa genes, promoters and terminators are listed in Additional file 1: Table S1. The cocoa genes were amplified from the synthesized genes; Promoter $\mathrm{P}_{T E F 1}$ of Ashbya gossypii was amplified from template pUG60 (Goldstein et al. 1999); Promoters $\mathrm{P}_{P G K 1}$ and $\mathrm{P}_{F B A 1}$, and terminators $\mathrm{T}_{A D H 1}, \mathrm{~T}_{G A T 2}$ and $\mathrm{T}_{C Y C 1}$ were amplified from genomic DNA of $\mathrm{S}$. cerevisiae CEN.PK 113-11C. The backbone fragment of plasmid pBS01A was amplified from the expression vector of pBS01A (derived from pSP-GM1, see the Additional file 1) (Chen et al. 2012). Promoters, cocoa genes and terminators were fused into cocoa gene expression cassettes using overlap extension PCR (Zhou et al. 2012). The gene expression cassettes were verified by PCR and the structure of all the cassettes is described in Fig. 2. The verified gene expressing cassettes were ligated into the amplified backbone fragment of plasmid pBS01A using the Gibson assembly method (NEB) to construct cocoa gene expression plasmids, which were verified with PCR and Sanger sequencing (Additional file 1: Tables S1, S2). Finally, the pBS01 plasmid and the plasmids harboring cocoa genes were used to transform S. cerevisiae CEN.PK 113-11C, to construct 15 new yeast strains (Table 1 ).

\section{$20 \mathbf{~ m l}$ shake flask cultivation and fatty acid analysis} $20 \mathrm{ml}$ shake flask fermentations were carried out in minimal medium (Verduyn et al. 1992) supplemented with $100 \mathrm{mg} \mathrm{l}^{-1}$ histidine. Three clones of each strain verified with PCR were used to inoculate $14 \mathrm{ml}$ sterile falcon tubes containing $2 \mathrm{ml}$ minimal medium and cultivated at $30{ }^{\circ} \mathrm{C}$ and $200 \mathrm{rpm}$ for $24 \mathrm{~h}$. The precultures were used to inoculate $100 \mathrm{ml}$ shake flask with $20 \mathrm{ml}$ fresh minimal medium at an initial OD600 $\mathrm{nm}$ of 0.1 that were then cultivated for $72 \mathrm{~h}$ at $30{ }^{\circ} \mathrm{C}$ and $200 \mathrm{rpm}$. The cultures were collected in $50 \mathrm{ml}$ falcon tubes and harvested by centrifugation at $3000 \mathrm{~g}$ for $10 \mathrm{~min}$. The collected yeast cells were washed once with distilled water and dried using a freezer dryer (Alpha 2-4 LSC, Christ GmbH). Finally, $10 \mathrm{mg}$ freeze-dried yeast biomass of each strain was used for fatty acid methyl ester (FAME) analyses using a microwave-assisted method (Khoomrung et al. 2012).

\section{I shake flask batch cultivation and lipid analysis}

In order to obtain enough lipids, 11 yeast biomass of three selected yeast strains harboring cocoa genes and one yeast strain harboring pBS01A were collected and used for lipid extraction, respectively. Two clones of 


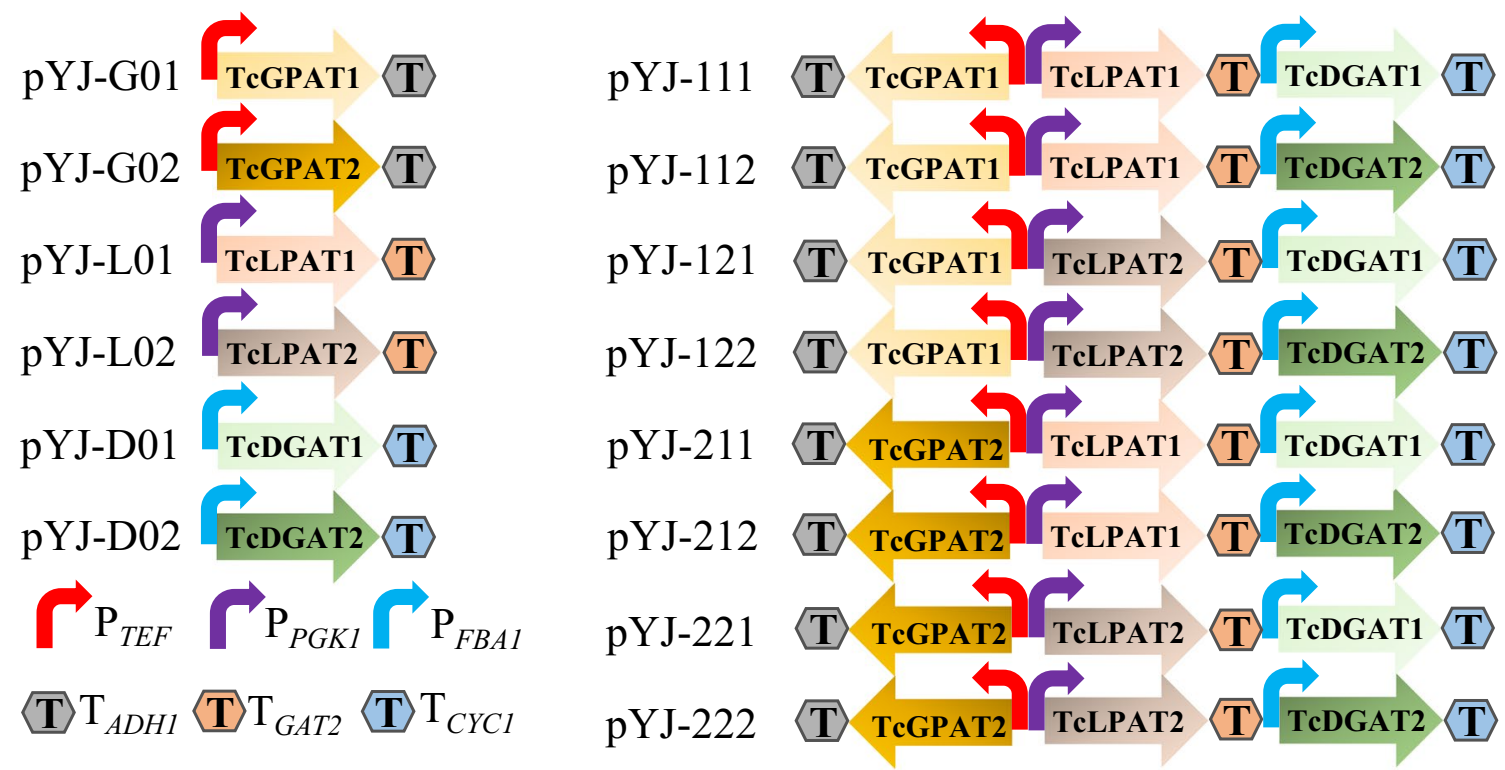

Fig. 2 Schematic organization of cocoa gene expression cassettes in each of the expression plasmids

each strain verified by PCR were used to inoculate $20 \mathrm{ml}$ fresh SC-URA medium in $50 \mathrm{ml}$ falcon tubes and cultivated at $30^{\circ} \mathrm{C}$ and $200 \mathrm{rpm}$ for $24 \mathrm{~h}$. Then the cultures of each strain were collected by centrifugation at $3000 \mathrm{~g}$ for $10 \mathrm{~min}$ and resuspended in $50 \mathrm{ml}$ fresh NLM medium. The resuspended yeast cells were used to inoculate $5 \mathrm{l}$ shake flask with $1 \mathrm{l}$ fresh NLM medium at an initial OD600 nm of 0.1 , and cultivated at $30{ }^{\circ} \mathrm{C}$ and $200 \mathrm{rpm}$ for $120 \mathrm{~h}$. The yeast cells were harvested by centrifuging at $6000 \mathrm{~g}$ for $15 \mathrm{~min} .30 \mathrm{ml}$ yeast cultures were collected separately and dried using a freezer dryer. The remaining yeast cells collected from 11 yeast biomass were used for lipid extraction. The collected yeast cells were washed once with distilled water before further use. $10 \mathrm{mg}$ of freeze dried yeast biomass was used for lipid extraction using a microwave-assisted methods followed by lipid analysis with HPLC-CAD (Khoomrung et al. 2013). The wet yeast biomass was used for large scale lipid extraction (Nambou et al. 2014; Yu et al. 2015). The lipid samples extracted from each strain were used for TAG analysis. TAGs in the lipids were measured by UPLC using RI detection, and the TAG compositions were expressed in relative area percentages (Shukla et al. 1983).

\section{Results}

Phylogenetic analysis of annotated cocoa GPAT, LPAT and DGAT genes revealed six potential CB biosynthetic genes

Usually, many TAG biosynthetic genes (GPAT, LPAT and DGAT genes) would be identified in one plant species, e.g. more than ten genes were annotated as GPAT genes in T. cacao (Argout et al. 2011; Motamayor et al. 2013). However, among all the cocoa genes annotated as GPAT, LPAT and DGAT genes, the ones actually responsible for CB biosynthesis are unknown (Argout et al. 2011; Motamayor et al. 2013). All amino acid sequences of annotated cocoa GPAT, LPAT and DGAT genes in the CGD and KEGG databases were downloaded and assigned the names TcGPAT1 to TcGPAT13, TcLPAT1 to TcLPAT10, TcDGAT1 to TcDGAT11, respectively. To identify potential CB biosynthetic genes, all annotated cocoa genes were compared with their corresponding reference genes of $A$. thaliana, $H$. sapiens and S. cerevisiae (Kanehisa et al. 2016). As GPAT, LPAT and DGAT genes of S. cerevisiae had been characterized and their function had been determined before, cocoa genes most similar to GPAT, LPAT and DGAT genes of $S$. cerevisiae might be functional in S. cerevisiae and they were prioritized for expression (Benghezal et al. 2007; Oelkers et al. 2002; Zheng and Zou 2001). In addition, genes which are similar to characterized GPAT, LPAT and DGAT genes of $A$. thaliana were also prioritized for expression.

Since all cocoa GPAT sequences were distinct from the two S. cerevisiae GPAT sequence of SCT1 and GPT2, the potential cocoa $\mathrm{CB}$ biosynthetic GPAT sequences were selected by comparison with the GPAT sequences of $A$. thaliana (Fig. 3a). As TcGPAT12 has $63.9 \%$ identity with ATS1 of A. thaliana, which might not be involved in TAG biosynthetic pathway, it probably is not a potential CB biosynthetic GPAT gene (Chen et al. 2011; Nishida et al. 1993; Nuccio and Thomas 1999). GPAT1, $-4,-5,-6$ and -7 of A. thaliana had been demonstrated to have GPAT 
activity (Zheng et al. 2003), and GPAT4, -6 and -8 of $A$. thaliana strongly preferred C16:0 and C18:1 $\omega$-oxidized acyl-CoAs over other substrates (Yang et al. 2012). TcGPAT1 has 81.8 and $82.8 \%$ identities with GPAT4 and GPAT8 of A. thaliana, respectively, and might be one potential CB biosynthetic GPAT gene (Fig. 3a). GPAT9 of $A$. thaliana was shown to be the ER-localized GPAT enzyme which is believed to be responsible for TAG biosynthesis (Shockey et al. 2016), and TcGPAT2 had 88.6\% identity with GPAT9 of $A$. thaliana (Fig. 3a), suggesting TcGPAT2 is one potential CB biosynthetic GPAT gene (Fig. 3a).

Though some annotated cocoa genes are very similar to the characterized LPAT genes of A. thaliana, three annotated cocoa LPAT sequences (TcLPAT1, TcLPAT2 and TCLPAT8) were very similar to the $S$. cerevisiae LPAT Slc1p (Benghezal et al. 2007; Nagiec et al. 1993), indicating these three cocoa genes might be the potential CB biosynthetic LPAT genes (Fig. 3b). As TcLPAT2 and TcLPAT 8 have $95.5 \%$ identity, and consist of 310 and 200 amino acids, respectively, TcLPAT2 is more likely to be a CB biosynthetic LPAT gene than TcLPAT8, because the most similar yeast LPAT sequence Slc1p has 303 amino acids and TcLPAT8 might be a gene fragment of TcLPAT2.

For DGAT, two cocoa DGAT sequences, TcDGAT1 and TcDGAT2, are very similar to Dga1p of S. cerevisiae and a DGAT of $A$. thaliana (Katavic et al. 1995; Oelkers et al. 2002; Routaboul et al. 1999; Sorger and Daum 2002; Zou et al. 1999); while other cocoa DGAT sequences (TcDGAT3-TcDGAT11) are more similar to Lrolp of $S$. cerevisiae or wax ester synthase of $A$. thaliana, hinting that they might have DGAT activity, but not necessarily represent CB biosynthetic DGAT genes (Fig. 3c). Besides, TcDGAT1 and TcDGAT2 had been characterized before, the results indicated that they displayed DGAT activity. By combining the phylogenetic analysis and the enzyme activity analysis, it was hypothesized that TcDGAT1 and TcDGAT2 were the potential CB biosynthetic DGAT genes.

Two cocoa GPAT genes (TcGPAT1 and TcGPAT2), two cocoa LPAT genes (TCLPAT1 and TcLPAT2) and two cocoa DGAT genes (TcDGAT1 and TcDGAT2), which are potentially responsible for $\mathrm{CB}$ biosynthesis, were therefore selected for codon optimization synthesis and expression in yeast based on this phylogenetic analyses.

\section{Expression of cocoa genes in S. cerevisiae changed its total fatty acid production}

The synthesized cocoa genes and cocoa gene combinations were assembled in expression cassettes using strong constitutive promoters and ligated into plasmid pBS01A, which resulted in 14 different plasmids (Additional file 1:
Table S2; Fig. 2). Plasmid pBS01A and the 14 other plasmids harboring cocoa genes were introduced into $S$. cerevisiae, generating the control strain YJ0 and another 14 yeast strains, respectively (Table 1 ). The fatty acid production and composition of each yeast strains were measured after cultivation in shake flasks. The relative $\mathrm{C} 16$ and $\mathrm{C} 18$ content of all the 15 different yeast strains were more than $97.3 \%$, which is consistent with previous studies that main fatty acids of S. cerevisiae are C16 and C18 (Khoomrung et al. 2012; Suutari et al. 1990). Compared with the control strain YJ0, fatty acid production of most yeast strains harboring cocoa genes increased (Fig. 4). Among the yeast strains harboring single cocoa genes, YJ-G01, YJ-G02 and YJ-L01 produced more total fatty acids than YJ0; YJ-D01 produced approximately the same amount of total fatty acids as YJ0; YJ-L02 and YJ-D01 produced less total fatty acids than YJ0. For the yeast strains harboring combinations of cocoa genes, YJ-112, -121, -122, -211, -212 and -221 produced more total fatty acids than YJ0, and YJ-111 and YJ-222 produced less total fatty acids than YJ0 (Fig. 4).

Due to variations between clones, among all the 14 yeast strains harboring cocoa genes, only YJ-212 (harboring the combination of TcGPAT2, TcLPAT1 and TcDGAT2) and YJ-221 (harboring the combination of TcGPAT2, TcLPAT2 and TcDGAT1) displayed a significant increase (29.1 and 9.9\%, respectively) of the total fatty acid amount compared to YJ0 (Fig. 4). Moreover, C16:1, C18:0 and C18:1 contents of YJ-212, and C16:0, C18:0 and C18:1 contents of YJ-221 were increased compared with YJ0. The C16:1. C18:0 and C18:1 content of YJ-212 was 21.0, 54.0 and 36.2\% higher compared with YJ0; and the C16:0, C18:0 and C18:1 content of YJ-221 was 41.2, 41.1 and $17.8 \%$ higher compared with YJ0 (Additional file 1: Table S3). In addition, the relative C16:0, C16:1, C18:0 and C18:1 contents of YJ-212 showed no significant difference compared with YJ0, but the relative C16:0, C16:1 and C18:0 contents of YJ-221 displayed a significant increase compared with the corresponding relative content of YJ0 (Additional file 1: Table S3). These results suggested that YJ-221 might be better for CBL production than YJ-212.

\section{Expression of cocoa gene combinations in S. cerevisiae altered lipid production and compositions}

In order to further investigate the effects of cocoa gene expression on lipid and TAG production of $S$. cerevisiae, three yeast strains harboring cocoa genes, YJ-111, -121 and -221 , and the control strain YJ0 were selected for lipid and TAG analyses. YJ-111 produced less fatty acids than YJ0; YJ-121 produced approximately the same amount of total fatty acids as YJ0; whereas YJ-221 produced more fatty acids than YJ0 and showed differences 

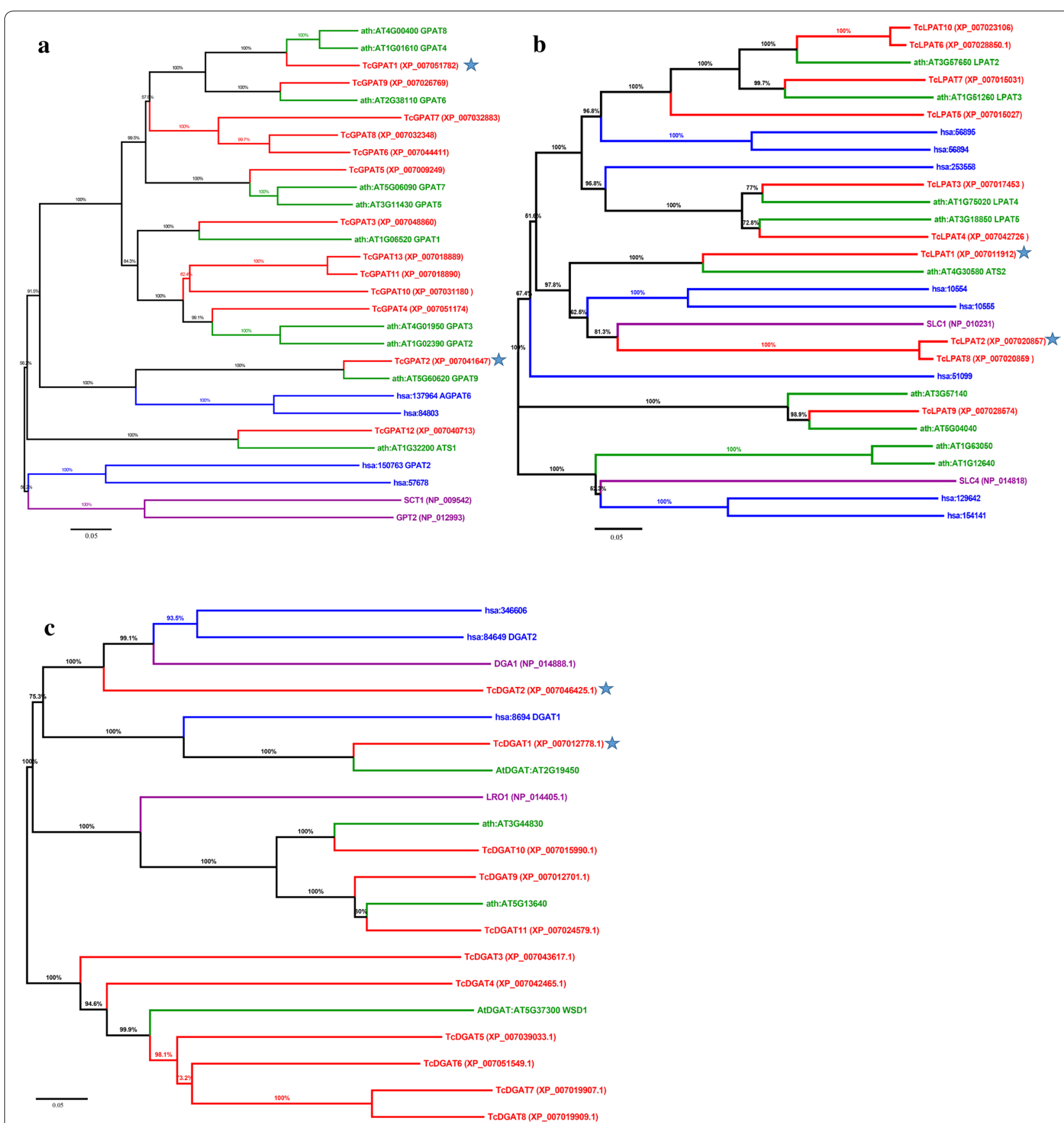

Fig. 3 Phylogenetic analysis of GPAT (a), LPAT (b) and DGAT (c) genes of T. cacao genomes with an unrooted tree. All neighbor-joining trees were constructed using the MEGA 6.06 software (bootstrap values: 1000) with the peptide sequences. The sequences of T. cacao are labeled in red; the sequences of $A$. thaliana are labeled in green; the sequences of $S$. cerevisiae are labeled in purple; the sequences of $H$. sapiens are labeled in blue. The GPAT sequences of T. cacao were named TcGPAT1 to TCGPAT13; the LPAT sequences of $T$. cacao were named TCLPAT1 to TCLPAT10; the DGAT sequences of T. cacao were named TCDGAT1 to TCDGAT11. Cocoa genes selected for synthesis are marked with asterisks. The bootstrap values are marked above the nodes and the scale bar is indicated under each tree

on $\mathrm{C} 18: 0$ and $\mathrm{C} 18: 1$ production and relative C18:0 content compared with YJ0 (Additional file 1: Table S3).

Total lipid profiles in yeasts usually cover TAGs, steryl esters (SE), ergosterol (ES), cardiolipin (CL), phosphatidic acid (PA), phosphatidylethanolamine (PE), phosphatidylinositol (PI), phosphatidylserine (PS), and phosphatidylcholine (PC) (Czabany et al. 2007; de Kroon et al. 2013; Kaneko et al. 1976). Though YJ-111 and YJ-121 did 
not display differences in total fatty acid production compared with YJ0 (Fig. 4), YJ-111 displayed differences compared with YJ0 in ES, PE, PC and PS production, YJ-121 showed differences compared with YJ0 in PE, PC and PS levels, suggesting the cocoa genes have an effect on yeast phospholipid production. YJ-221, which displayed differences with YJ0 in the total fatty acid content, also exhibited differences compared to YJ0 in TAG production (Fig. 5). In fact, YJ-221 produced 2.25-fold more TAG than YJ0. Though YJ-221 exhibited enhanced accumulation of TAGs, no other lipids of YJ-221 displayed differences compared with YJ0 (Fig. 5). Besides, while the TAG content comprised $37.2 \%$ of the total lipids in YJ0, TAGs represented 48.0, 56.3 and $60.3 \%$ of the total lipids in YJ-111, -121 and -221 (Fig. 5).

\section{Expressing cocoa gene combinations in S. cerevisiae increased potential CBL composition and production}

Though the methods employed in this study do not allow to determine the exact position of each fatty acid within a TAG molecule, the fatty acid composition of each TAG can be determined. All four yeast strains tested in this study produced at least 22 different kinds of TAGs, and there was a major change in the TAG composition following expression of the cocoa genes (Fig. 6; Additional file 1: Figure S1). Most of the TAGs accounted for less than 5\% of the total TAG pool (Additional file 1: Figure S1). Concerning CBL TAGs, potential POP (C16:0, C18:1, C16:0) and potential POS (C16:0, C18:1, C18:0), YJ-111, -121 and -221 displayed differences compared with YJ0; the proportion of potential POP in YJ-111, -121 and -221

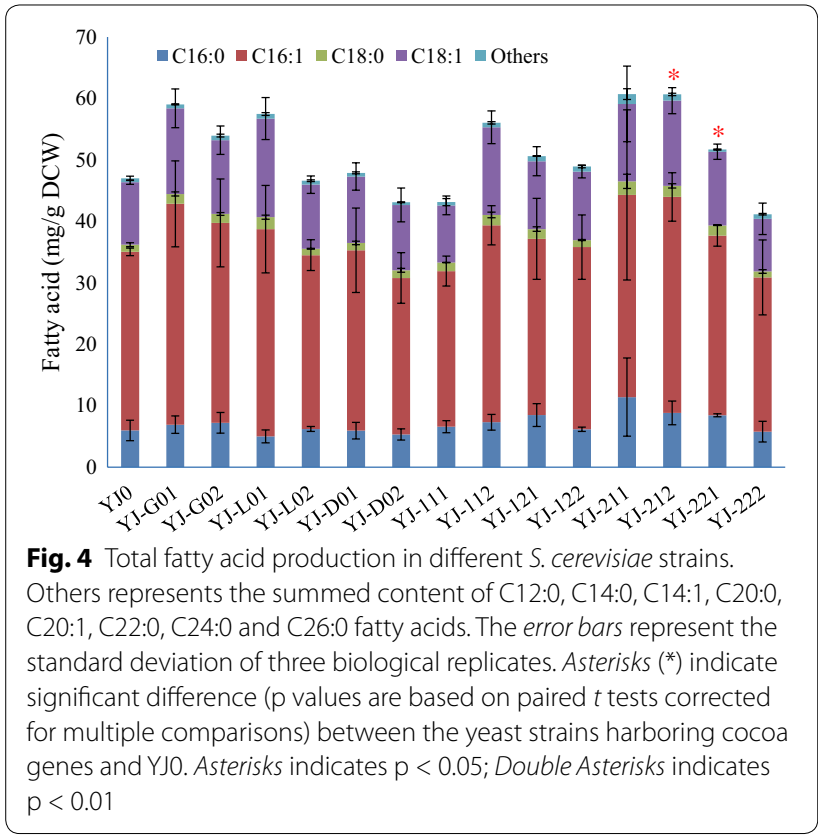

increased by 185, 197 and 177\%, respectively, while the proportion of potential POS increased by 183, 222 and $187 \%$, respectively (Fig. 6). For another CBL TAG, potential SOS (C18:0, C18:1, C18:0), YJ-121 and YJ-221 also displayed a significant difference compared with YJ0. In fact, the potential SOS proportion had increased from $0.14 \%$ in YJ0 to $0.81 \%$ in YJ- 121 and $0.64 \%$ in YJ-221, which means an increase of 476 and 354\%, respectively (Fig. 6). The increase of potential CBL components, from $1.63 \%$ in $\mathrm{YJ} 0$ to $4.72 \%$ in $\mathrm{YJ}-111,5.38 \%$ in $\mathrm{YJ}-121$ and $4.82 \%$ in $\mathrm{YJ}-221$ suggests that the cocoa genes are functional in S. cerevisiae. As YJ-221 also produces 2.25fold more TAGs compared with YJ0, its potential CBL production was 6.7-fold improved compared with YJ0, showing that the combination of TcGPAT2, TcLPAT2 and TcDGAT1 (YJ-221) not only increased TAG production of $S$. cerevisiae, but also allowed $S$. cerevisiae to accumulate more potential CBL.

\section{Fatty acid profiles and compositions of the TAGs}

In order to gain insight into substrate preferences of GPAT, LPAT and DGAT of S. cerevisiae under physiological conditions, the relative fatty acid composition of all TAGs was analyzed. The main fatty acids in the TAGs in these four yeast strains were $\mathrm{C} 16$ and $\mathrm{C} 18$ fatty acids, which is consistent with the total fatty acid composition results (Fig. 7). Generally, saturated fatty acids were increased in the TAGs of YJ-111, -121 and -221, which would be beneficial for CBL biosynthesis as there are more saturated fatty acids than unsaturated fatty acids in CB (Jahurul et al. 2013). In detail, the C16:0 proportion in TAGs of YJ-111, -121 and -221 was increased compared with YJ0, while the $\mathrm{C} 16: 1$ proportion was reduced. Of the three engineered yeast strains, only YJ-221 exhibited a significant decrease in the C18:1 proportion. Also the C18:0 fatty acid ratio in the TAGs was increased for all the 3 yeast strain harboring cocoa genes, however, this increase was not significant, showing that it is necessary to screen additional cocoa GPAT, LPAT and DGAT genes in order to increase the incorporation of C18:0 into TAGs.

\section{Discussion}

In this study, we aimed at increasing CBL production in $S$. cerevisiae by expressing genes of $T$. cacao potentially involved in CBL biosynthesis. There are at least 13 putative GPAT, 10 LPAT and 11 DGAT genes in T. cacao. By combining published information on the cocoa genome and a phylogenetic approach (Argout et al. 2011; Motamayor et al. 2013), we identified two GPAT, two LPAT and two DGAT genes of $T$. cacao, which might be responsible for $\mathrm{CB}$ biosynthesis. Expressing selected single cocoa genes or cocoa gene combinations (one GPAT, 


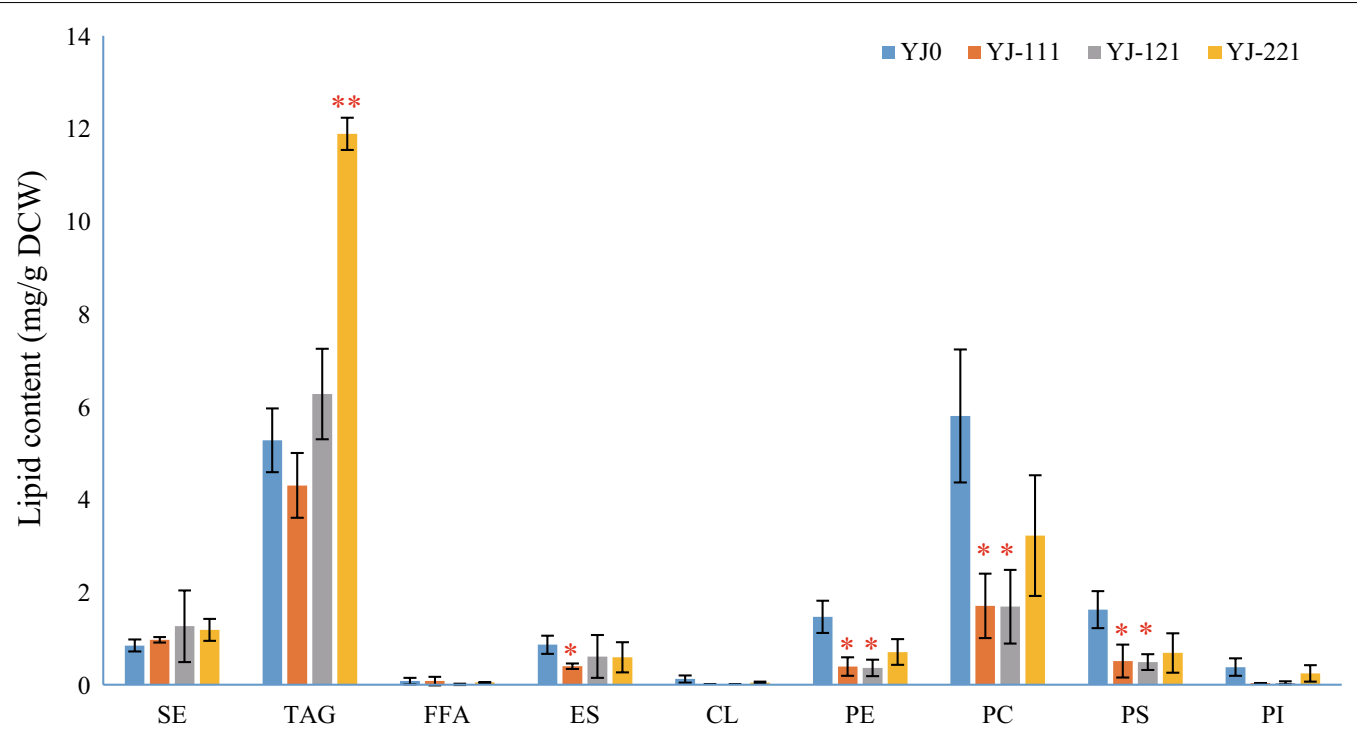

Fig. 5 Total lipid production in different S. cerevisiae strains. SE steryl esters, FFA free fatty acids, ES ergosterol, CL cardiolipin, PE phosphatidylethanolamine, $P C$ phosphatidylcholine, PS phosphatidylserine, $P$ I phosphatidylinositol. Phosphatidic acids were not determined in this study. The error bars represent the standard deviation of two biological replicates. Asterisks $\left(^{*}\right)$ indicate significant difference ( $p$ values are based on paired $t$ tests corrected for multiple comparisons) between the yeast strains harboring cocoa genes and YJ0. Asterisks indicates $p<0.05$; Double Asterisks indicates $p<0.01$

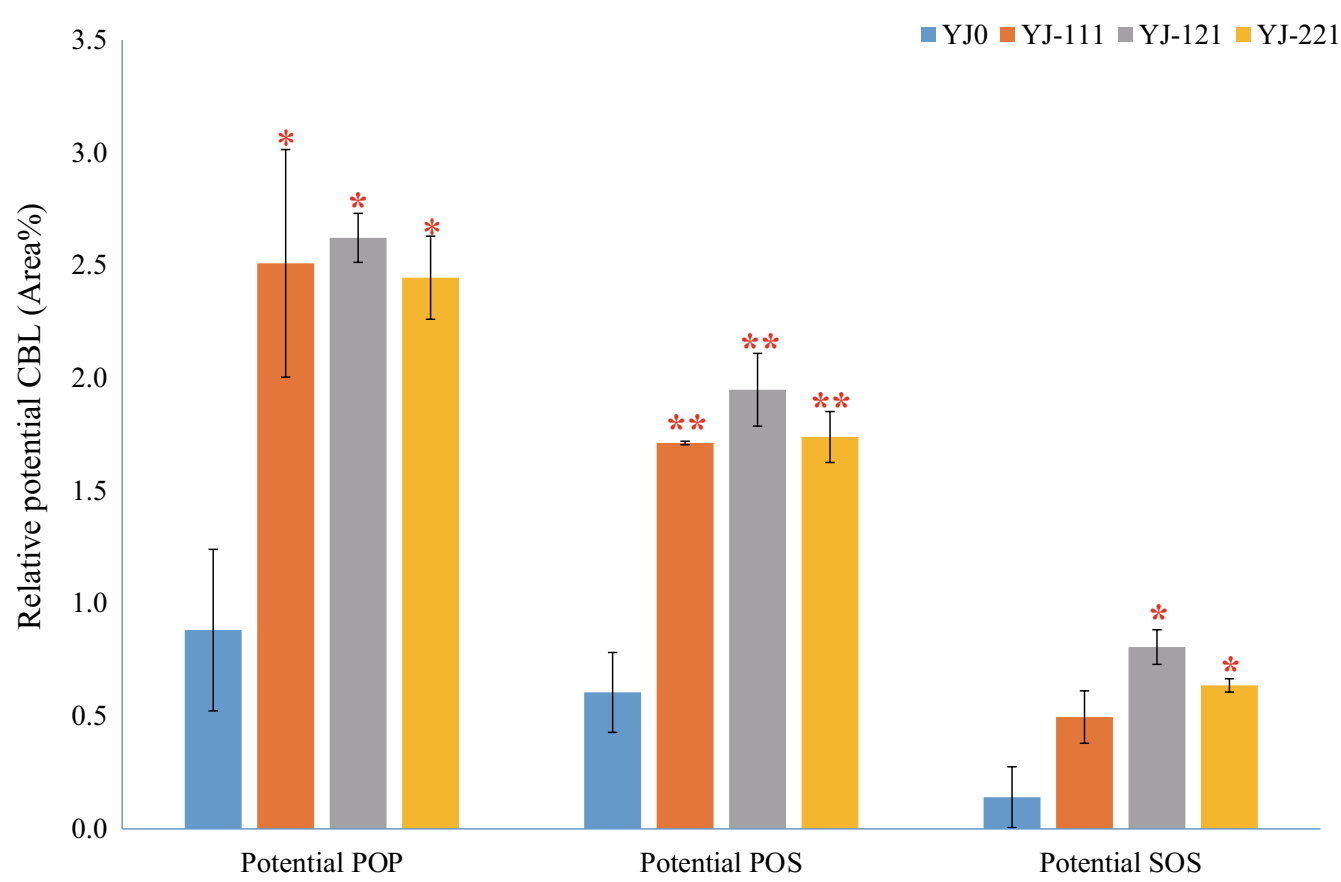

Fig. 6 Relative potential CBL content of S. cerevisiae strains. The error bars represent the standard deviation of two biological replicates. Asterisks (*) indicate significant differences ( $p$ values are based on paired $t$ tests corrected for multiple comparisons) between the yeast strains harboring cocoa genes and YJ0. Asterisks indicates $p<0.05$; Double Asterisks indicates $p<0.01$

one LPAT and one DGAT gene) in S. cerevisiae had an effect on total fatty acid production in this study. Especially, expression of cocoa gene combinations was able to significantly alter the total fatty acid production in yeast, enabling some yeast strains to produce more CBL, indicating that the selected cocoa genes played important 


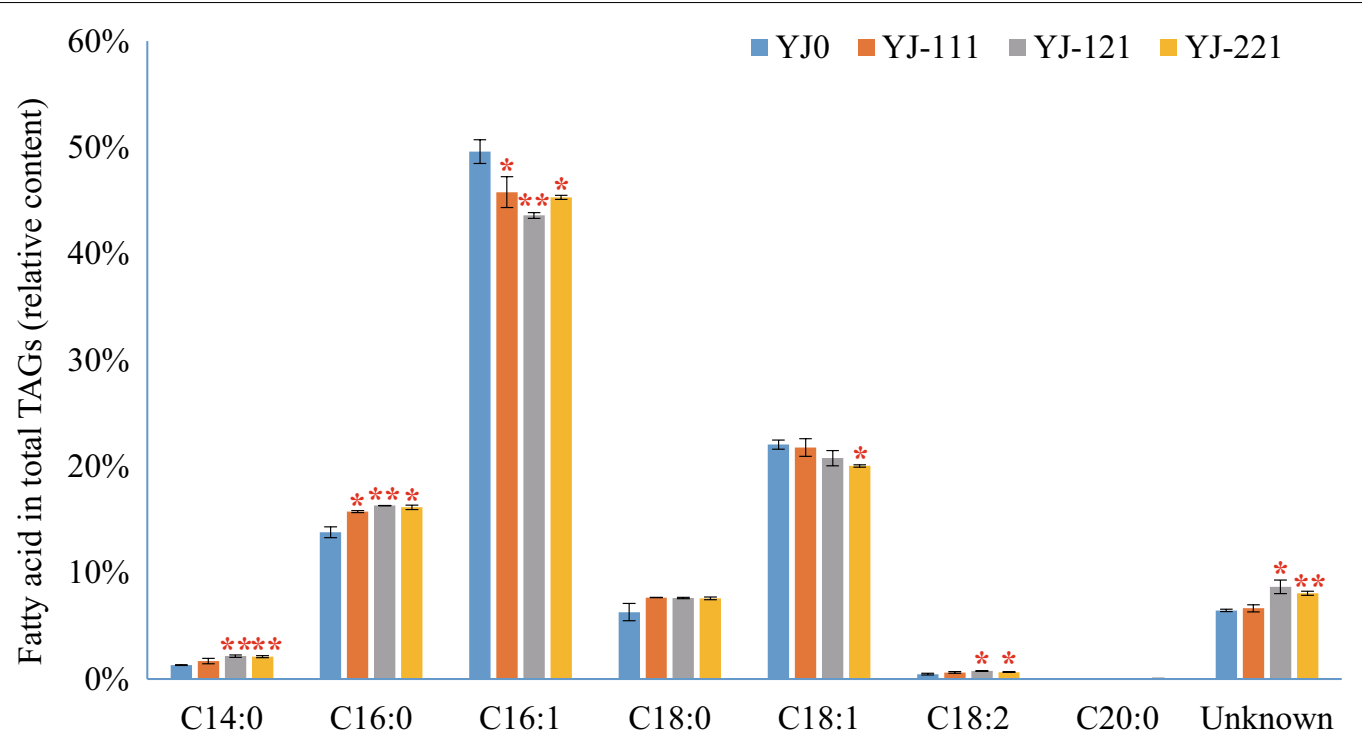

Fig. 7 Relative fatty acid composition of the TAGs of each S. cerevisiae strain. The error bars represent the standard deviation of two biological replicates. Asterisks $\left(^{*}\right)$ indicate significant differences ( $p$ values are based on paired $t$ tests corrected for multiple comparisons) between the yeast strains harboring cocoa genes and YJO. Asterisks indicates $p<0.05$; Double Asterisks indicates $p<0.01$

roles in CBL production in S. cerevisiae. As all the six cocoa genes were selected based on combining published information on the cocoa genome and a phylogenetic analysis, it is a clear example that phylogenetic analysis can be used for gene or pathway mining for metabolic engineering (Mak et al. 2015).

Though CBL production is determined by three different enzymes (Coleman and Lee 2004), expressing a single DGAT gene in S. cerevisiae can increase total TAG production (Bouvier-Navé et al. 2000; Runguphan and Keasling 2014). Besides, deletion or overexpression of GPAT or LPAT of S. cerevisiae can alter total lipid production and composition (Benghezal et al. 2007; Zheng and Zou 2001). By comparing the three engineered yeast strains YJ-111, YJ-121 and YJ-221 with YJ0, we showed that lipid and TAG composition of these three yeast strains harboring cocoa genes were different from YJ0, suggesting expression of some GPAT, LPAT and DGAT genes could increase yeast TAG production and the cocoa gene combinations functioned in S. cerevisiae. The potential CBL production of the three yeast strains, especially, potential SOS production drastically increased, indicating the selected cocoa genes are promising candidate CB biosynthetic genes.

Compared with the fact that there are 14-16.4\% POP, 34.6-38.3\% POS and $23.7-28.4 \%$ SOS in CB (Lipp and Anklam 1998), the CBL content in the engineered S. cerevisiae strains obtained in this study is less than $5.4 \%$, demonstrating that more efforts are need to improve
CBL production in yeast for further industrial application. Considering that YJ-221 was the only of the three yeast strains that showed significant difference with YJ0 in the C18:1 proportion of TAGs and that the remaining 11 cocoa GPAT and 8 cocoa LPAT genes not selected in this study might also have effects on CBL biosynthesis (Argout et al. 2011; Motamayor et al. 2013), suggests that expressing some of these in S. cerevisiae might improve $\mathrm{CBL}$ production and reveal more candidate genes for yeast CBL production. In addition, many strategies have been implemented for increasing fatty acid or TAG production, such as overexpression of fatty acid or TAG biosynthesis genes of DGA1, acetyl-CoA carboxylase, fatty acid synthase (Kamisaka et al. 2007; Runguphan and Keasling 2014; Zhou et al. 2016), and these could also be used for further increasing CBL production in $S$. cerevisiae.

In conclusion, we increased CBL production by $S$. cerevisiae through expressing selected genes of T. cacao potentially involved in $\mathrm{CB}$ biosynthesis, which might be used in yeast CBL production in future. Additionally, our approach of integrating plant genome data screening and metabolic engineering may also find application in production of other value-added plant metabolites using $S$. cerevisiae as a cell factory.

\section{Additional file}

Additional file 1. Additional tables and figure. 


\section{Abbreviations}

CB: cocoa butter; TAG: triacylglycerol; POP: 1,3-dipalmitoyl-2-oleoylglycerol (C16:0-C18:1-C16:0); POS: 1-palmitoyl-3-stearoyl-2-oleoyl-glycerol (C16:0-C18:1-C18:0); SOS: 1,3-distearoyl-2-oleoyl-glycerol (C18:0-C18:1C18:0); CBL: CB-like lipids; GPAT: glycerol-3-phosphate acyltransferase; LPAT: lysophospholipid acyltransferase; DGAT: diacylglycerol acyltransferase; acyl-CoA: acyl-coenzyme A; DAG: diacylglycerol; CG: Cacao Genome Database; SE: steryl esters; ES: ergosterol; CL: cardiolipin; PA: phosphatidic acid; PE: phosphatidylethanolamine; PI: phosphatidylinositol; PS: phosphatidylserine; PC: phosphatidylcholine.

\section{Authors' contributions}

JN and VS conceived the study. YW designed and performed most of the experiments; MG and DB designed and performed part of the experiments. YW wrote the manuscript; JN, VS, MG and DB edited the manuscript. All authors read and approved the final manuscript.

\section{Author details}

${ }^{1}$ Department of Biology and Biological Engineering, Chalmers University of Technology, SE-41296 Gothenburg, Sweden. ${ }^{2}$ Novo Nordisk Foundation Center for Biosustainability, Chalmers University of Technology, SE-41296 Gothenburg, Sweden. ${ }^{3}$ Novo Nordisk Foundation Center for Biosustainability, Technical University of Denmark, 2800 Kongens Lyngby, Denmark.

\section{Acknowledgements}

This work was funded by AAK AB, the Knut and Alice Wallenberg Foundation and the Novo Nordisk Foundation. We thank Berit Kristensen, AAK A/S, for performing the TAG analysis, and Morten Emil Møldrup, AAK AB, for scientific discussions during the project. We thank Julia Karlsson, Jacob Kindbom and Raphael Ferreira for their help in GC and HPLC analyses. We thank Anastasia Krivoruchko for providing the pBS01 plasmid.

\section{Competing interests}

The authors declare that they have no competing interests.

\section{Availability of data and materials}

The dataset supporting the conclusions of this article is included within the article and its supplementary files.

\section{Funding}

This work was funded by $A A K A B$, the Knut and Alice Wallenberg Foundation and the Novo Nordisk Foundation.

Received: 20 January 2017 Accepted: 27 January 2017

Published online: 06 February 2017

\section{References}

Argout X, Salse J, Aury J-M, Guiltinan MJ, Droc G, Gouzy J, Allegre M, Chaparro C, Legavre T, Maximova SN (2011) The genome of Theobroma cacao. Nat Genet 43(2):101-108

Benghezal M, Roubaty C, Veepuri V, Knudsen J, Conzelmann A (2007) SLC1 and SLC4 encode partially redundant acyl-coenzyme A 1-acylglycerol-3-phosphate O-acyltransferases of budding yeast. J Biol Chem 282(42):30845-30855

Bouvier-Navé P, Benveniste P, Oelkers P, Sturley SL, Schaller H (2000) Expression in yeast and tobacco of plant CDNAs encoding acyl CoA: diacylglycerol acyltransferase. Eur J Biochem 267(1):85-96

Chapman KD, Ohlrogge JB (2012) Compartmentation of triacylglycerol accumulation in plants. J Biol Chem 287(4):2288-2294

Chen X, Snyder CL, Truksa M, Shah S, Weselake RJ (2011) sn-Glycerol-3-phosphate acyltransferases in plants. Plant Signal Behav 6(11):1695-1699

Chen Y, Partow S, Scalcinati G, Siewers V, Nielsen J (2012) Enhancing the copy number of episomal plasmids in Saccharomyces cerevisiae for improved protein production. FEMS Yeast Res 12(5):598-607

Clough Y, Faust H, Tscharntke T (2009) Cacao boom and bust: sustainability of agroforests and opportunities for biodiversity conservation. Conserv Lett 2(5):197-205
Coleman RA, Lee DP (2004) Enzymes of triacylglycerol synthesis and their regulation. Prog Lipid Res 43(2):134-176

Czabany T, Athenstaedt K, Daum G (2007) Synthesis, storage and degradation of neutral lipids in yeast. Biochim Biophys Acta 1771(3):299-309

de Kroon Al, Rijken PJ, De Smet CH (2013) Checks and balances in membrane phospholipid class and acyl chain homeostasis, the yeast perspective. Prog Lipid Res 52(4):374-394

Ejsing CS, Sampaio JL, Surendranath V, Duchoslav E, Ekroos K, Klemm RW, Simons K, Shevchenko A (2009) Global analysis of the yeast lipidome by quantitative shotgun mass spectrometry. Proc Natl Acad Sci 106(7):2136-2141

Entian K-D, Kötter P (2007) 25 Yeast genetic strain and plasmid collections. Method Microbiol 36:629-666

Goldstein AL, Pan X, McCusker JH (1999) Heterologous URA3MX cassettes for gene replacement in Saccharomyces cerevisiae. Yeast 15(6):507-511

Jahurul M, Zaidul I, Norulaini N, Sahena F, Jinap S, Azmir J, Sharif K, Omar AM (2013) Cocoa butter fats and possibilities of substitution in food products concerning cocoa varieties, alternative sources, extraction methods, composition, and characteristics. J Food Eng 117(4):467-476

Kamisaka Y, Tomita N, Kimura K, Kainou K, Uemura H (2007) DGA1 (diacylglycerol acyltransferase gene) overexpression and leucine biosynthesis significantly increase lipid accumulation in the $\Delta s n f 2$ disruptant of Saccharomyces cerevisiae. Biochem J 408:61-68

Kanehisa M, Sato Y, Kawashima M, Furumichi M, Tanabe M (2016) KEGG as a reference resource for gene and protein annotation. Nucleic Acids Res 44(D1):D457-D462

Kaneko H, Hosohara M, Tanaka M, Itoh T (1976) Lipid composition of 30 species of yeast. Lipids 11(12):837-844

Katavic V, Reed DW, Taylor DC, Giblin EM, Barton DL, Zou J, Mackenzie SL, Covello PS, Kunst $L$ (1995) Alteration of seed fatty acid composition by an ethyl methanesulfonate-induced mutation in Arabidopsis thaliana affecting diacylglycerol acyltransferase activity. Plant Physiol 108(1):399-409

Katoh K, Standley DM (2013) MAFFT multiple sequence alignment software version 7: improvements in performance and usability. Mol Biol Evol 30(4):772-780

Khoomrung S, Chumnanpuen P, Jansa-Ard S, Nookaew I, Nielsen J (2012) Fast and accurate preparation fatty acid methyl esters by microwave-assisted derivatization in the yeast Saccharomyces cerevisiae. Appl Microbiol Biotechnol 94(6):1637-1646

Khoomrung S, Chumnanpuen P, Jansa-Ard S, Ståhlman M, Nookaew I, Borén J, Nielsen J (2013) Rapid quantification of yeast lipid using microwave-assisted total lipid extraction and HPLC-CAD. Anal Chem 85(10):4912-4919

Kim HU, LiY, Huang AH (2005) Ubiquitous and endoplasmic reticulumlocated lysophosphatidyl acyltransferase, LPAT2, is essential for female but not male gametophyte development in Arabidopsis. Plant Cell 17(4):1073-1089

Koch B, Schmidt C, Daum G (2014) Storage lipids of yeasts: a survey of nonpolar lipid metabolism in Saccharomyces cerevisiae, Pichia pastoris, and Yarrowia lipolytica. FEMS Microbiol Rev 38(5):892-915

Li Y, Beisson F, Koo AJ, Molina I, Pollard M, Ohlrogge J (2007) Identification of acyltransferases required for cutin biosynthesis and production of cutin with suberin-like monomers. Proc Natl Acad Sci 104(46):18339-18344

Li M, Kildegaard KR, Chen Y, Rodriguez A, Borodina I, Nielsen J (2015) De novo production of resveratrol from glucose or ethanol by engineered Saccharomyces cerevisiae. Metab Eng 32:1-11

Lipp M, Anklam E (1998) Review of cocoa butter and alternative fats for use in chocolate-part A. Compositional data. Food Chem 62(1):73-97

Mak WS, Tran S, Marcheschi R, Bertolani S, Thompson J, Baker D, Liao JC, Siegel JB (2015) Integrative genomic mining for enzyme function to enable engineering of a non-natural biosynthetic pathway. Nat Commun 6:10005

Motamayor JC, Mockaitis K, Schmutz J, Haiminen N, Livingstone D III, Cornejo O, Findley S, Zheng P, Utro F, Royaert S (2013) The genome sequence of the most widely cultivated cacao type and its use to identify candidate genes regulating pod color. Genome Biol 14:r53

Nagiec MM, Wells G, Lester R, Dickson R (1993) A suppressor gene that enables Saccharomyces cerevisiae to grow without making sphingolipids encodes a protein that resembles an Escherichia coli fatty acyltransferase. J Biol Chem 268(29):22156-22163 
Nambou K, Zhao C, Wei L, Chen J, Imanaka T, Hua Q (2014) Designing of a "cheap to run" fermentation platform for an enhanced production of single cell oil from Yarrowia lipolytica DSM3286 as a potential feedstock for biodiesel. Bioresour Technol 173:324-333

Nishida I, Tasaka Y, Shiraishi H, Murata N (1993) The gene and the RNA for the precursor to the plastid-located glycerol-3-phosphate acyltransferase of Arabidopsis thaliana. Plant Mol Biol 21(2):267-277

Nuccio ML, Thomas TL (1999) ATS1 and ATS3: two novel embryo-specific genes in Arabidopsis thaliana. Plant Mol Biol 39(6):1153-1163

Oelkers P, Cromley D, Padamsee M, Billheimer JT, Sturley SL (2002) The DGA1 gene determines a second triglyceride synthetic pathway in yeast. J Biol Chem 277(11):8877-8881

Ratledge C (2002) Regulation of lipid accumulation in oleaginous microorganisms. Biochem Soc Trans 30(6):1047-1049

Routaboul J-M, Benning C, Bechtold N, Caboche M, Lepiniec L (1999) The TAG1 locus of Arabidopsis encodes for a diacylglycerol acyltransferase. Plant Physiol Biochem 37(11):831-840

Runguphan W, Keasling JD (2014) Metabolic engineering of Saccharomyces cerevisiae for production of fatty acid-derived biofuels and chemicals. Metab Eng 21:103-113

Shockey J, Regmi A, Cotton K, Adhikari N, Bates PD (2016) Identification of Arabidopsis GPAT9 (At5g60620) as an essential gene involved in triacylglycerol biosynthesis. Plant Physiol 170(1):163-179

Shukla V, Schiøtz Nielsen W, Batsberg W (1983) A simple and direct procedure for the evaluation of triglyceride composition of cocoa butters by high performance liquid chromatography: a comparison with the existing TLC-GC method. Fett Wiss Technol 85(7):274-278

Sorger D, Daum G (2002) Synthesis of triacylglycerols by the acyl-coenzyme A: diacyl-glycerol acyltransferase Dga1p in lipid particles of the yeast Saccharomyces cerevisiae. J Bacteriol 184(2):519-524

Sorger D, Daum G (2003) Triacylglycerol biosynthesis in yeast. Appl Microbiol Biotechnol 61(4):289-299

Stukey JE, McDonough VM, Martin CE (1989) Isolation and characterization of OLE1, a gene affecting fatty acid desaturation from Saccharomyces cerevisiae. J Biol Chem 264(28):16537-16544

Stukey JE, McDonough VM, Martin CE (1990) The OLE1 gene of Saccharomyces cerevisiae encodes the delta 9 fatty acid desaturase and can be functionally replaced by the rat stearoyl-CoA desaturase gene. J Biol Chem 265(33):20144-20149

Suutari M, Liukkonen K, Laakso S (1990) Temperature adaptation in yeasts: the role of fatty acids. Microbiology 136(8):1469-1474
Tamura K, Stecher G, Peterson D, Filipski A, Kumar S (2013) MEGA6: molecular evolutionary genetics analysis version 6.0. Mol Biol Evol 30(12):2725-2729

Turchetto-Zolet AC, Maraschin FS, de Morais GL, Cagliari A, Andrade CM, Margis-Pinheiro M, Margis R (2011) Evolutionary view of acyl-CoA diacylglycerol acyltransferase (DGAT), a key enzyme in neutral lipid biosynthesis. BMC Evol Biol 11(1):263

Verduyn C, Postma E, Scheffers WA, Van Dijken JP (1992) Effect of benzoic acid on metabolic fluxes in yeasts: a continuous-culture study on the regulation of respiration and alcoholic fermentation. Yeast 8(7):501-517

Yang W, Simpson JP, Li-Beisson Y, Beisson F, Pollard M, Ohlrogge JB (2012) A land-plant-specific glycerol-3-phosphate acyltransferase family in Arabidopsis: substrate specificity, sn-2 preference, and evolution. Plant Physiol 160(2):638-652

Yang X, Jin G, Gong Z, Shen H, Song Y, Bai F, Zhao ZK (2014) Simultaneous utilization of glucose and mannose from spent yeast cell mass for lipid production by Lipomyces starkeyi. Bioresour Technol 158:383-387

Yu X, Dong T, Zheng Y, Miao C, Chen S (2015) Investigations on cell disruption of oleaginous microorganisms: hydrochloric acid digestion is an effective method for lipid extraction. Eur J Lipid Sci Tech 117(5):730-737

Zhang Y (2012) Recombinant expression of plant diacylglycerol acyltransferases from tissues that accumulate saturated fatty acids. Dissertation, University of Alberta, Edmonton

Zheng Z, Zou J (2001) The initial step of the glycerolipid pathway Identification of glycerol 3-phosphate/dihydroxyacetone phosphate dual substrate acyltransferases in Saccharomyces cerevisiae. J Biol Chem 276(45):41710-41716

Zheng Z, Xia Q, Dauk M, Shen W, Selvaraj G, Zou J (2003) Arabidopsis AtGPAT1, a member of the membrane-bound glycerol-3-phosphate acyltransferase gene family, is essential for tapetum differentiation and male fertility. Plant Cell 15(8):1872-1887

Zhou YJ, Gao W, Rong Q, Jin G, Chu H, Liu W, Yang W, Zhu Z, Li G, Zhu G (2012) Modular pathway engineering of diterpenoid synthases and the mevalonic acid pathway for miltiradiene production. J Am Chem Soc 134(6):3234-3241

Zhou YJ, Buijs NA, Zhu Z, Qin J, Siewers V, Nielsen J (2016) Production of fatty acid-derived oleochemicals and biofuels by synthetic yeast cell factories. Nat Commun 7:11709

Zou J, Wei Y, Jako C, Kumar A, Selvaraj G, Taylor DC (1999) The Arabidopsis thaliana TAG1 mutant has a mutation in a diacylglycerol acyltransferase gene. Plant J 19(6):645-653

\section{Submit your manuscript to a SpringerOpen ${ }^{\circ}$ journal and benefit from:}

- Convenient online submission

- Rigorous peer review

- Immediate publication on acceptance

- Open access: articles freely available online

- High visibility within the field

- Retaining the copyright to your article

Submit your next manuscript at springeropen.com 